\title{
Orientation-dependent crack-tip blunting and crack propagation in a single crystal $\mathrm{BCC}$ iron
}

\author{
SURAJIT KUMAR PAUL ${ }^{1, *}$, SUNIL KUMAR ${ }^{2}$ and S TARAFDER ${ }^{2}$ \\ ${ }^{1}$ Department of Mechanical Engineering, Indian Institute of Technology Patna, Bihta, Patna 801106, India \\ ${ }^{2}$ Division of Materials Engineering, CSIR-National Metallurgical Laboratory, Jamshedpur 831007, India \\ *Author for correspondence (paulsurajit@yahoo.co.in)
}

MS received 27 November 2017; accepted 19 January 2018; published online 3 December 2018

\begin{abstract}
Atomistic simulations of cracks with four different orientations in body-centered cubic single crystal iron are presented using molecular dynamics. Crystal orientation has considerable effect on the activation and evolution of crack propagation mechanisms. The results reveal that (a) crack-tip blunting depends on the crystallographic orientation, (b) continuous generation of dislocations form crack tip occurs for large crack-tip blunting, and (c) absence of deformation activities like dislocation generation, twin formation, etc. at the crack tip results in crack propagation in a brittle manner.
\end{abstract}

Keywords. Crack propagation; crack-tip blunting; molecular dynamics; crystal orientation; BCC iron.

\section{Introduction}

Fracture mechanics is normally used as a tool to understand the propagation and growth of cracks in crystalline materials. Crack propagation involves breaking of individual bonds between atoms at the atomic scale. It is intimately dependent on the local atomic environment, such as the type of atoms, crystal structure and lattice orientation. At the atomistic level, the physics of crack propagation will depend upon the crystal structure of the material, dislocation and slip activity primarily controlling crack propagation in single crystal metals, while additionally in polycrystalline metals, transgranular or intergranular crack paths competing in the crack-growth process.

In the last decade, a number of investigations were conducted at various length scales to understand the deformation and damage mechanisms leading to crack propagation. A number of continuum mechanics-based approaches, such as finite element method (FEM) [1-6], crystal plasticity finite element method (CPFEM) [7-9] and extended finite element method (XFEM) [10], were employed for understanding crack propagation at mesoscopic or macroscopic scales. Continuum mechanics-based approaches are not capable of providing an insight into fracture at the atomic scale and also are unsuitable for addressing the physics of the process. Thus, molecular dynamics (MD) has become one of the most widely used methods for investigating the fundamental mechanism of deformation and damage, and also the fracture behaviours of materials at the atomic scale [11-16].

$\alpha$-Iron is a body-centered cubic (BCC) metal, and for this type of structure, a number of important possible deformation mechanisms exists. Slip in BCC metal occurs in the closest packed $\langle 111\rangle$ directions. The most common slip planes for BCC metal are $\{110\},\{112\}$ and $\{123\}$ [17]. Under some special conditions like high deformation rate and low temperature, twinning can also take place in BCC metals on the $\{112\}$ planes in $\langle 111\rangle$ directions. In pure iron and low-alloy steels, it was experimentally established that the above slip systems are the most active $[18,19]$. Researchers have observed experimentally that $\{100\}$ planes are most commonly seen to cleave, whereas $\{110\},\{112\}$ and $\{123\}$ planes create cracks [20].

A number of MD simulations were conducted in various metals to understand crack propagation at the atomistic level. $\mathrm{Xu}$ and Deng [21] described the stress field around the crack tip and its variation during crack growth in single-crystal aluminium. Guo et al [22] reported the local stress at the crack tip and its effect on martensitic phase transformation. $\mathrm{Wu}$ and Yao [23] demonstrated the effect of temperature variation on crack propagation and on stress-field properties for single-crystal nickel. Shastry and Farkas [24] noticed that the crack extended in a brittle manner for [100] crack front of $\alpha$-iron, while a ductile response was observed for [110] crack front for cracks on a (110) plane at $0 \mathrm{~K}$ temperature. Using an N-body Finnis-Sinclair (F-S) potential, Hora et al [25] simulated two cracks of $\alpha$-iron on a (001) plane at the temperatures of 0 and $300 \mathrm{~K}$. They reported that the central cracks extend in a brittle manner at the temperature of $0 \mathrm{~K}$, while at the temperature of $300 \mathrm{~K}$, pronounced slip occurs. Similarly, Machová and Beltz [26] also observed crack extension by bond breaking along the original crack plane of (001) at $0 \mathrm{~K}$ temperature for $\alpha$-iron. They tested two different crack lengths at the same condition, the shortest crack produced twinning and the longest showed pure cleavage 
fracture. Zhang et al [27] investigated the mechanisms of crack propagation in single crystal, bicrystal and tricrystal nickels. They pointed out that the grain boundary plays an important role in the initiation and propagation of crack. They also noticed that void initiates at the triple junction of grain boundaries and contributes to accelerating the crack propagation for tricrystal. Zhang et al [28] showed through molecular dynamics simulation that the crack propagation is suppressed at the grain boundary above a critical twist angle between grain boundaries. They also reported that the higher energy in the grain boundary with larger twist angle contributes to facilitating the movement of the glissile dislocation along the grain boundary rather than across the grain boundary, which leads to the propagation of the crack along the grain boundary. Sung and Chen [29] did molecular dynamics simulation on single-crystal nickel to study the crack growth and propagation. They noticed that brittle to ductile transition occurs between temperatures of 50 and $700 \mathrm{~K}$, and the brittle fracture happens at lower temperature. They reported that for $\mathrm{Ni}$ nanoribbons, the critical fracture strain are in the order of $\langle 111\rangle>\langle 100\rangle>\langle 110\rangle$.

In the present investigation, the effect of orientations on the crack-propagation behaviour of $\alpha$-iron (BCC) single crystal is investigated using molecular dynamics employing Mendelev embedded atom method (EAM) potential [30]. BCC iron single crystal with crack-plane orientation of (001), (110), (111) and (11 $\overline{2})$ are considered in this study. The crackpropagation mechanisms during tensile loading are studied in BCC iron single crystal with various crack orientations.

\section{MD simulation details}

All MD simulations were performed in large scale atomic/ molecular massively parallel simulator (LAMMPS) package [31] utilizing an EAM potential for BCC iron given by Mendelev and co-workers [30]. This potential is based on the framework of a Finnis-Sinclair $(\mathrm{F}-\mathrm{S})$ type body potential. Deformation behaviour of nanoscale material is dependent on its size. The careful selection of size must be selected to achieve meaningful results as the size of atomistic deformation simulation is limited because of computational time. In the current MD simulation study, an initial dimension of $60 a \times 16 a \times 60 a$, where $a=2.855 \AA$ ( $a$ is lattice parameter of $\mathrm{BCC}$ iron) was selected for $\mathrm{BCC}$ iron single crystal with different orientations. The simulation box contains about 110,000 iron atoms arranged in BCC lattice. Periodic boundary condition is selected only along the thickness axis (y-axis), while the width direction ( $\mathrm{x}$ axis) is kept free. Tensile loading is applied in the length direction (z-axis). To examine the orientation-dependence crack-propagation mechanism in BCC iron single crystal, different crack plane orientations of (001), (110), (1111) and $(1 \overline{1} \overline{2})$ are considered. Figure 1 shows the initial crystal orientations and crack orientations of the BCC iron single crystal. The initial crack is created by removing the atoms.

Energy minimization is performed by conjugate gradient (CG) method for initial construction of the single crystal. Velocity Verlet algorithm is utilized to integrate the equation of motion with a time step of $1 \mathrm{fs}$. The stable structure thus, obtained is thermally equilibrated to a temperature of $300 \mathrm{~K}$. After completion of the equilibration process, the single crystals are deformed at a constant engineering strain rate of $10^{-8} \mathrm{~s}^{-1}$ with respect to initial box size in the length $(\mathrm{z})$ direction. Constant NVE (constant number of atoms, constant volume, constant energy) is used to simulate crack propagation in BCC iron single crystal. Due to the inherent time-scale limitations in MD simulations, the selected strain rate is much higher than typical experimental strain rate. The adaptive common neighbour analysis (a-CNA) method [32,33] is used to visualize defects in the crystalline phases. In the present work, face-centered cubic (FCC), BCC and non-structured atoms (often existing at the interface or at the dislocation core) are coloured green, blue and white, respectively. Visualization tool (OVITO) [34] is used for visualization and analysis of deformation mechanism (types of dislocation and twin).

\section{Results and discussion}

Crack propagation can begin in multiple modes in the atomic lattices like ductile mode due to void nucleation, growth and coalescence, brittle mode because of cleavage, or a mixture of the brittle and ductile modes. The crack-geometry evolution and crack propagation are the prime interest of investigation in this work. Presence of any defect near crack tip is not included in this simulation, however, it can affect simulation results.

\subsection{Orientations on the (001) crack plane}

Up to 0.065 strain (figure $2 \mathrm{~d}$ ), the width of notch increases with the increase in strain due to plastic deformation. It can be said that the crack is arrested in a certain extent at early stage of deformation as little notch blunting is observed. With increase in plastic deformation, a fresh crack initiates from the tip of the partly blunted notch at a strain of 0.0725 (figure 2e). At strain of 0.08 (figure $2 \mathrm{f}$ ), fast propagation of crack is noticed. The crack plane orientation of (001) also shows formation of FCC structure, the first sign of FCC formation is noticed at a strain of 0.0525 (figure $2 b$ ). In this case, the FCC region is placed at $\{101\}$ plane i.e., $35.3^{\circ}$ with respect to the crack plane, and no twinning is detected. Initially, the FCC region extends in length and after that in width also with increase in straining. Difficulty in dislocation slip for this crystal orientation is thought to be the cause of FCC formation. Ersland et al [14] also noticed the formation of FCC region at crack tip for the crack-plane orientation of (001) in BCC iron. They also observed that the FCC regions grow until the crack starts growing, and they continue to grow further as they follow the crack tip through the material. 
(a)
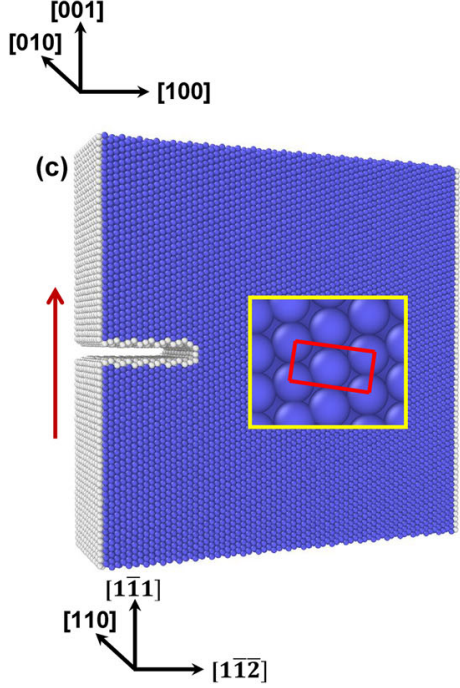

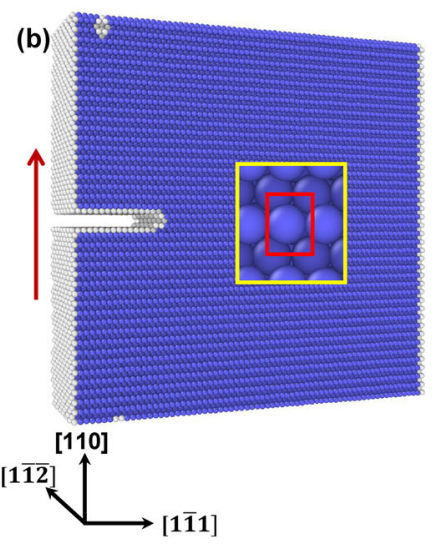

(d)

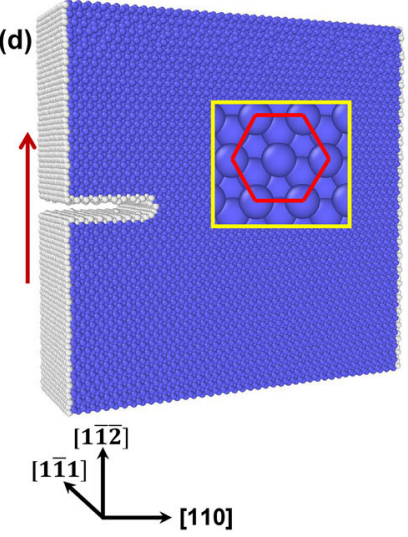

Figure 1. The initial orientations of $\alpha$-iron (BCC) with lateral cracks in

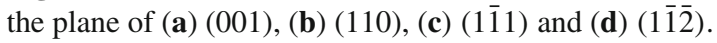
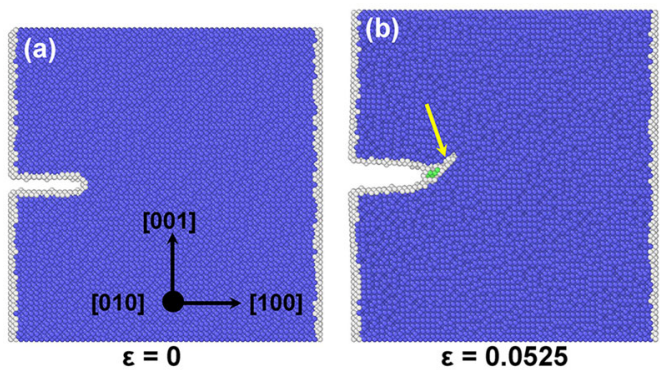

$\varepsilon=0.0525$

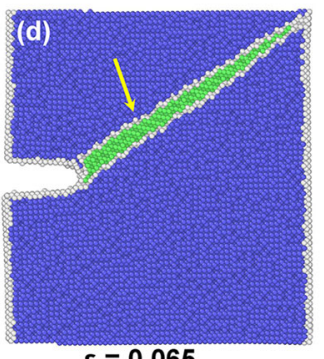

$\varepsilon=0.065$

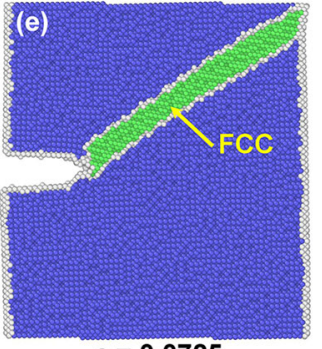

$\varepsilon=0.0725$

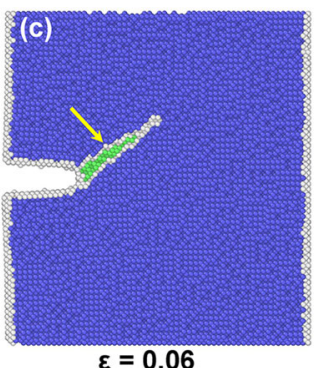

$\varepsilon=0.06$

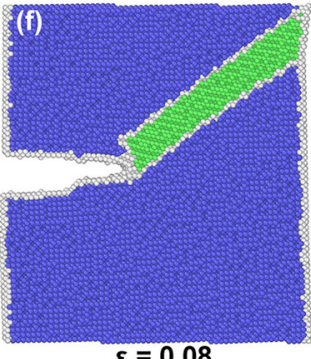

$\varepsilon=0.08$

Figure 2. A through-thickness cracks simulated in the (001) plane. During tensile straining, FCC structure formation at the tip of crack. Atoms are coloured due to CNA, where blue is BCC, green is FCC, and white is an unknown structure. 


\subsection{Orientations on the (110) crack plane}

For the crack plane orientation of (110), edge dislocations with Burgers vector of 1/2(111) started emitting from the beginning of plastic deformation. Figure $3 \mathrm{~b}$ shows emission of edge dislocation at a strain of 0.0475 . Figure 3 also shows that the crack tip emits edge dislocation at angles $\pm 54.7^{\circ}$ continuously with increase in strain. No fresh crack initiation is noticed up to the strain of 0.5 and crack tip gets blunted to a large extent. Also, no void nucleation is noticed at the strain of 0.5 , because the nucleation sites like grain/phase boundary, precipitate, second phase, etc. are absent in pure single crystal BCC iron. No FCC phase formation or twinning is observed in this orientation. Sainath et al [15] also reported for $\langle 110\rangle$

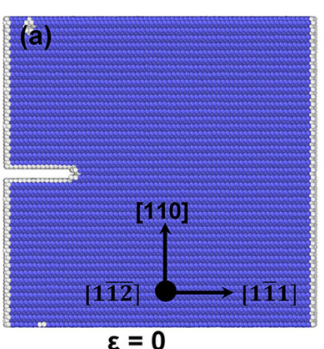

$\varepsilon=0$

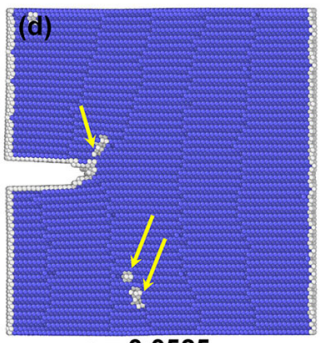

$\varepsilon=0.0525$

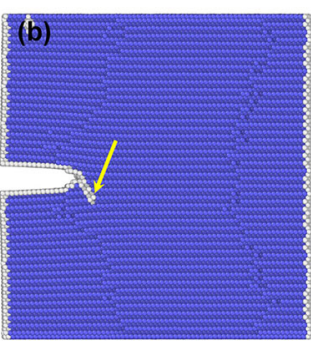

$\varepsilon=0.0475$

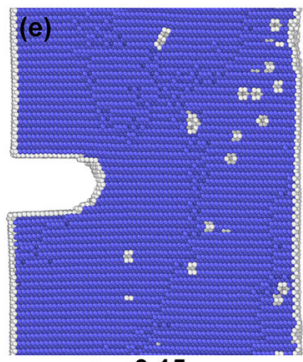

$\varepsilon=0.15$
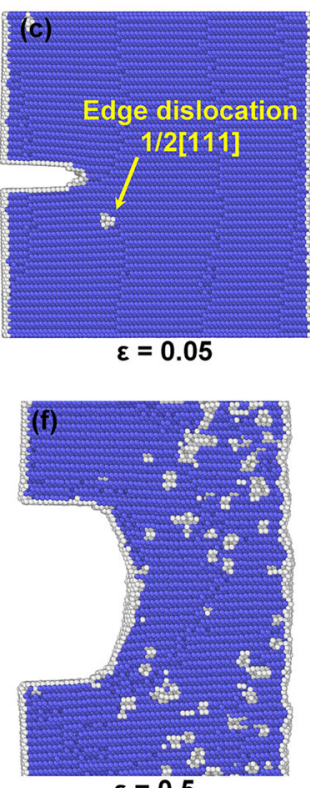

$\varepsilon=0.5$

Figure 3. A through-thickness cracks simulated in the (110) plane. During tensile straining, emission of edge dislocation with Burgers vector of 1/2[111] from the crack tip. Atoms are coloured due to CNA, where blue is BCC, and white is an unknown structure.
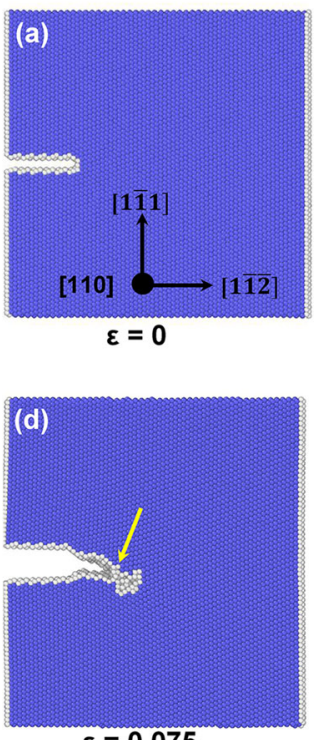

$\varepsilon=0.075$

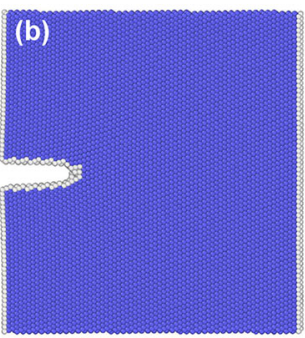

$\varepsilon=0.0525$

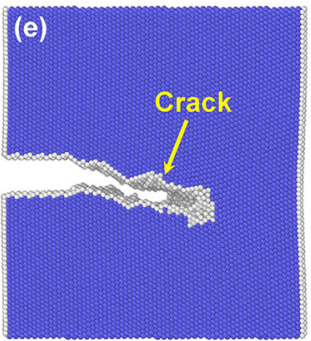

$\varepsilon=0.0825$

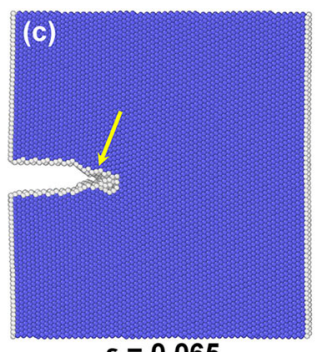

$\varepsilon=0.065$

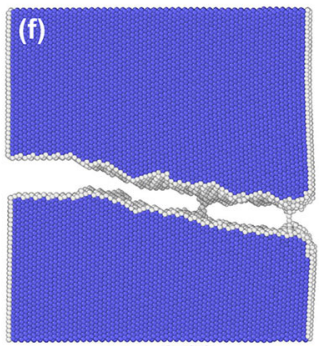

$\varepsilon=0.09$

Figure 4. A through-thickness cracks simulated in the (011) plane. Almost brittle-type crack propagation. Atoms are coloured due to CNA, where blue is $\mathrm{BCC}$, and white is an unknown structure. 

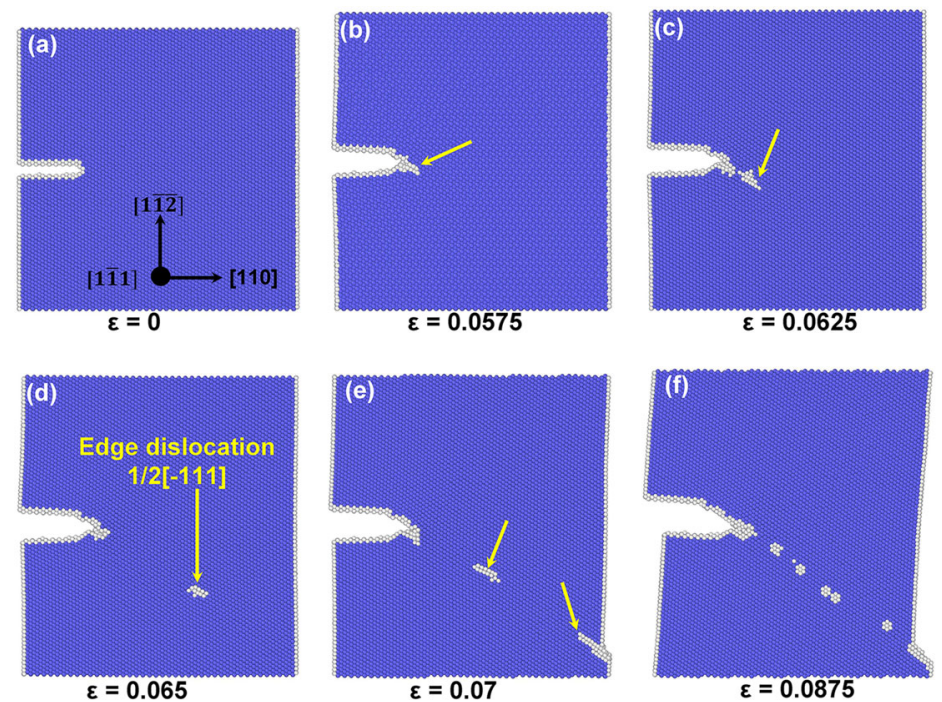

Figure 5. A through-thickness cracks simulated in the (1 $\overline{1} \overline{2})$ plane. During tensile straining, emission of edge dislocation with Burgers vector of $1 / 2[\overline{1} 11]$ from the crack tip. Atoms are coloured due to CNA, where blue is BCC, and white is an unknown structure.

nanowire, the yielding occurred by the nucleation of multiple $1 / 2\langle 111\rangle$ dislocation loops from the corner of the nanowire at a strain level of $14.4 \%$. Ersland et al [14] also reported emission of edge dislocations in the $\{112\}\langle 111\rangle$ slip system from the tip of crack with same orientation.

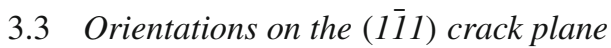

No dislocation emission, twin formation and FCC phase formation are noticed for the crack-plane orientation of (11i1). No crack-tip blunting is also noticed for this crack-plane orientation. After initial elastic deformation, the crack started to propagate at around $12.5^{\circ}$ angle in a brittle manner. Figure 4 shows that within very short straining, the full single crystal gets fractured and separated into two halves.

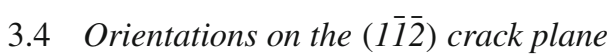

Edge dislocations with Burgers vector of $1 / 2(1 \overline{1} 1)$ are emitted from the beginning of plastic deformation for the crack plane orientation of $(1 \overline{1} \overline{2})$. Emission of edge dislocation at the strain of 0.0575 is shown in figure $5 b$. For the crack plane orientation of ( $(1 \overline{1} \overline{2})$ mode II (in-plane shear) type of deformation is observed, while mode I (opening) type of deformation is detected for the crack-plane orientation of (110). The crack tip releases edge dislocation continuously at angles $\pm 54.7^{\circ}$ with increasing deformation (figure 5). In this crack orientation, no FCC phase formation or twinning is noticed.

From the above four crack plane orientations, (110) orientation is most ductile, huge crack-tip blunting takes place and mode I (opening) type of deformation occurs, while (1111) orientation is most brittle, no crack-tip blunting occurs and crack propagates rapidly.

\section{Conclusions}

Molecular dynamic simulations were performed using an EAM potential on BCC single crystal iron with four different orientations of crack planes. The crack planes (001), (110), $(1 \overline{1} 1)$ and $(1 \overline{1} \overline{2})$ were selected and loaded in opening mode. The following conclusions can be made for this atomistic simulation study:

Crack-tip blunting can be used as a parameter to assess ductility. Crack-tip blunting depends on the orientation of crack plane. Four crack planes can be graded on crack-tip blunting in the decreasing order of $(110)>(1 \overline{1} \overline{2})>(001)>$ (111). Massive crack-tip blunting occurs and mode I (opening) type of deformation takes place for crack orientation of (110), while no crack-tip blunting happens and crack propagates rapidly for crack orientation of (11)1).

Crack-tip blunting is controlled by dislocation activity, twin formation and phase change at crack tip. Absence of any such activity results in crack propagation in a brittle manner. Continuous emission of dislocation is observed for crack orientation of (110), while no dislocation emission, twin formation and other phase formation are noticed for crack orientation of $(1 \overline{1} 1)$.

Crystal orientation has significant effect on crack-tip deformation mechanism, crack-tip blunting and finally, crack propagation in $\mathrm{BCC}$ iron.

\section{References}

[1] Özden U A, Mingard K P, Zivcec M, Bezold A and Broeckmann C 2015 Int. J. Refract. Met. Hard Mater. 49261 
[2] Dewang Y, Hora M S and Panthi S K 2015 Trans. Nonferr. Met. Soc. China 252308

[3] Paul S K and Tarafder S 2013 Int. J. Press. Vessel. Pip. 101 81

[4] Paul S K 2016 Eng. Fract. Mech. 15990

[5] Paul S K 2016 Eng. Fract. Mech. 15813

[6] Paul S K 2016 Theor. Appl. Fract. Mech. 84183

[7] Proudhon H, Li J, Wang F, Roos A, Chiaruttini V and Forest S 2016 Int. J. Fatigue 82238

[8] Lin B, Zhao L G and Tong J 2011 Eng. Fract. Mech. 78 2174

[9] Li L, Shen L and Proust G 2015 Mech. Mater. 8184

[10] Keyhani A, Goudarzi M, Mohammadi S and Roumina R 2015 Comput. Mater. Sci. 10498

[11] White P 2012 Int. J. Fatigue 44141

[12] Sainath G, Choudhary B K and Jayakumar T 2015 Comput. Mater. Sci. 10476

[13] Kumar S and Das S K 2017 Phys. Chem. Chem. Phys. https:// doi.org/10.1039/C7CP03902F

[14] Ersland C H, Vatne I R and Thaulow C 2012 Model. Simul. Mater. Sci. Eng. 20075004

[15] Sainath G and Choudhary B K 2016 Comput. Mater. Sci. 111 406

[16] Zhang Y, Jiang S, Zhu X and Zhao Y 2016 Eng. Fract. Mech. 168147

[17] Honeycombe R W K 1968 The plastic deformation of metals (London: Edward Arnold)
[18] Allen N P, Hopkins B E and McLennan J E 1956 Proc. R. Soc. Lond. A 234221

[19] Biggs W D and Pratt P L 1958 Acta Metall. 6694

[20] Nohava J, Haŭsild P, Karlík M and Bompard P 2002 Mater. Charact. 49211

[21] Xu S and Deng X 2008 Nanotechnology 19115705

[22] Guo Y F, Wang Y S, Zhao D L and Wu W P 2007 Acta Mater. 556634

[23] Wu W P and Yao Z Z 2012 Theor. Appl. Fract. Mech. 62 67

[24] Shastry V and Farkas D 1996 Model. Simul. Mater. Sci. Eng. 4 473

[25] Hora P, Pelikán V, Machová A, Spielmannová A, Prahl J, Landa M et al 2008 Eng. Fract. Mech. 753612

[26] Machová A and Beltz G E 2004 Mater. Sci. Eng. A 387-389 414

[27] Zhang Y, Jiang S, Zhu X and Zhao Y 2017 Res. Phys. 7 1722

[28] Zhang Y, Jiang S, Zhu X and Zhao Y 2017 Physica E 87281

[29] Sung P-H and Chen T-C 2015 Comput. Mater. Sci. 102151

[30] Mendelev M I, Han S, Srolovitz D J, Ackland G J, Sun D Y and Asta M 2003 Philos. Mag. 833977

[31] Plimpton S J 1995 J. Comput. Phys. 1171

[32] Faken D and Jónsson H 1994 Comput. Mater. Sci. 2279

[33] Duo L I, Wang F C, Yang Z Y and Zhao Y P 2014 Sci. China Phys. Mech. 572177

[34] Stukowski A 2010 Model. Simul. Mater. Sci. Eng. 1861 